\title{
Effect of Reynolds and Prandtl Numbers on Mixed Convection in an Obstructed Vented Cavity
}

\author{
M. M. Rahman ${ }^{1 *}$, M. M. Billah' ${ }^{2}$, and M. A. Alim ${ }^{1}$ \\ ${ }^{1}$ Department of Mathematics, Bangladesh University of Engineering and Technology (BUET), \\ Dhaka-1000, Bangladesh \\ ${ }^{2}$ Department of Arts and Sciences, Ahsanullah University of Science and Technology (AUST), \\ Dhaka-1208, Bangladesh
}

Received 7 February 2010, accepted in final revised form 22 January 2011

\begin{abstract}
A numerical investigation is conducted to analyze the steady flow and thermal fields as well as heat transfer characteristics in a vented square cavity with a built-in heat conducting horizontal solid circular obstruction. Hydrodynamic behavior, thermal characteristics and heat transfer results are obtained by solving the couple of Navier-Stokes and energy equations by using a weighted residuals Finite element method. The computation was made for different Reynolds number, Prandtl number ranging from 50 to 200 and from 0.71 to 7.1 at the three different convective regimes. Three different regimes are observed with increasing Ri: forced convection (with negligible free convection), mixed convection (comparable free and forced convection) and free convection dominated region (with higher free convection). The results are presented to show the effects of the Reynolds number, Prandtl number on flow pattern, thermal field and heat transfer characteristics at the three convective regimes. It is found that the flow and thermal field strongly depend on the Reynolds number, Prandtl number as well as Richardson number. As the Reynolds number and Prandtl number increase, the heat transfer rate increases but average fluid temperature in the cavity and temperature at the cylinder center decrease at the three convective regimes.

Keywords: Mixed convection; Finite element method; Obstructed vented cavity; Prandtl number.
\end{abstract}

(c) 2011 JSR Publications. ISSN: 2070-0237 (Print); 2070-0245 (Online). All rights reserved.

doi:10.3329/jsr.v3i2.4344 J. Sci. Res. 3 (2), 271-281 (2011)

\section{Introduction}

Mixed convection in enclosures is encountered in many engineering systems such as cooling of electronic components, ventilation in building and fluid movement in solar energy collectors etc. An investigation of above phenomena incorporating a heat conducting solid cylinder extends its application in many other realistic situations. For example, any projection on a motherboard of a computer and a conductive material in an

* Corresponding author: mmustafizurrahman@math.buet.ac.bd 
inert atmosphere inside a furnace with a constant flow of gas from outside constitute a practical application for the present study.

House et al. [1] investigated the effect of a square heat conducting body on natural convection in a vertical enclosure. They found that heat transfer across the cavity enhanced by a body with a thermal conductivity ratio less than unity. Later on, Oh et al. [2] studied the same geometry numerically, where the conducting body generated heat within the cavity. They concluded that the flow was driven by a temperature difference across the cavity. Roychowdhury et al. [3] analyzed the natural convective flow and heat transfer features for a heated body placed inside a square enclosure with different thermal boundary conditions. Dong and Li [4] performed the conjugate effect of natural convection and conduction in a complicated enclosure. They concluded that the flow and heat transfer increased with the increase of thermal conductivity in the solid region, and both the geometric shape and Rayleigh number also affected the overall flow and heat transfer greatly. Bilgen and Yamane [5] examined numerically the effect of conjugate heat transfer by laminar natural convection and conduction in two-dimensional rectangular enclosures with openings. They investigated the effects of the various geometrical parameters and the thickness of the insulation layer on the fluid flow and heat transfer characteristics. Bhoite et al. [6] studied numerically the mixed convection flow and heat transfer in a shallow enclosure with a series of block-like heat generating components for a range of Reynolds numbers and Grashof numbers and block-to-fluid thermal conductivity ratios. They showed that higher Reynolds numbers created a recirculation region of increasing strength at the core region and the effect of buoyancy became insignificant beyond a Reynolds number of typically 600 and hence the thermal conductivity ratio had a negligible effect on the velocity fields. Braga and de Lemos [7] investigated laminar natural convection within a square cavity filled with a fixed volume of conducting solid material consisting of either circular or square obstacles. They presented that the average Nusselt number for cylindrical rods was slightly lower than those for square rods. The problem of laminar natural convection heat transfer in a square cavity with an adiabatic arc shaped baffle was numerically analyzed by Tasnim and Collins [8]. These authors identified that flow, and thermal fields were modified by the blockage effect of the baffle and the degree of flow modification due to blockage was enhanced by increasing the shape parameter of the baffle. The conjugate natural convection heat transfer inside an inclined square cavity with an internal conducting block was carried out by Das and Reddy [9]. At the same time, Xu et al. [10] have experimentally demonstrated the thermal flow around a square obstruction on a vertical wall in a differentially heated cavity. Zhao et al. [11] numerically investigated the conjugate natural convection in enclosures with external and internal heat sources.

Literature review shows a number of investigations on natural convection heat transfer in a cavity along with a heat conducting body. To the best of the authors' knowledge, limited work has been done in a vented enclosure containing a solid circular cylinder. The main objective of the present study is to examine the effect of Reynolds number and Prandtl number on fluid flow and heat transfer in a ventilated square cavity. 


\section{Physical Model}

Fig. 1 shows the geometry of the present work. The problem deals with a heat conducting solid circular cylinder with a diameter $d$ and thermal conductivity $k_{s}$ located at the center of a square ventilated cavity with sides of length $L$. The right vertical wall of the square cavity is placed at the constant temperature $T_{h}$ but other walls of the cavity are kept adiabatic. A cartesian co-ordinate system is used with the origin at the lower left hand corner of the computational domain. The inlet port is located at the bottom of the left vertical wall while the outlet port is positioned at the top of the right wall. However, the size of the two openings is assumed as the one-tenth of the cavity length $(w=0.1 \mathrm{~L}$ ). Air is considered as fluid. Cold fluid flows through the inlet at a uniform velocity $u_{i}$. In addition, the incoming flow is at the ambient temperature $T_{i}$ and the outgoing flow is assumed to have zero diffusion flux for all variables. All solid boundaries are assumed to be rigid no-slip walls.

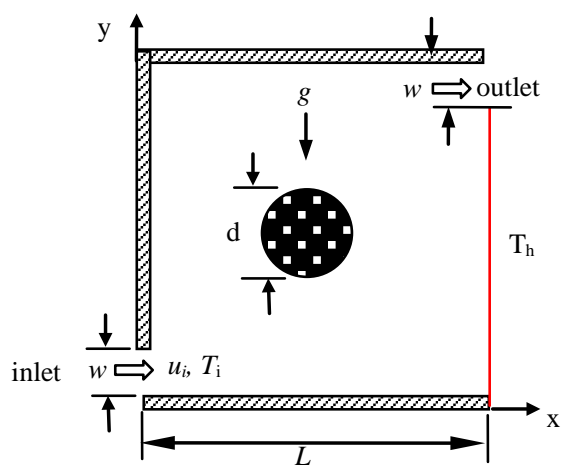

Fig. 1. Schematic of the problem with the domain and boundary conditions.

\section{Mathematical Model}

The governing equations describing the problem under consideration are based on the laws of mass, linear momentum and energy with buoyancy forces. The energy equation is written using the Boussinesq approximation. This means that all thermo-physical properties of the fluid at a reference temperature are taken constant except in the buoyancy term of the momentum equation. In addition, the radiation heat exchange is negligible in this study. The non-dimensional governing equations can be written as: (see [12]):

$$
\frac{\partial U}{\partial X}+\frac{\partial V}{\partial Y}=0
$$




$$
\begin{aligned}
& U \frac{\partial U}{\partial X}+V \frac{\partial U}{\partial Y}=-\frac{\partial P}{\partial X}+\frac{1}{R e}\left(\frac{\partial^{2} U}{\partial X^{2}}+\frac{\partial^{2} U}{\partial Y^{2}}\right) \\
& U \frac{\partial V}{\partial X}+V \frac{\partial V}{\partial Y}=-\frac{\partial P}{\partial Y}+\frac{1}{R e}\left(\frac{\partial^{2} V}{\partial X^{2}}+\frac{\partial^{2} V}{\partial Y^{2}}\right)+R i \theta \\
& U \frac{\partial \theta}{\partial X}+V \frac{\partial \theta}{\partial Y}=\frac{1}{\operatorname{RePr}}\left(\frac{\partial^{2} \theta}{\partial X^{2}}+\frac{\partial^{2} \theta}{\partial Y^{2}}\right)
\end{aligned}
$$

For solid cylinder, the energy equation is

$$
\frac{\partial^{2} \theta_{s}}{\partial X^{2}}+\frac{\partial^{2} \theta_{s}}{\partial Y^{2}}=0
$$

The non-dimensional variables used in the above equations are defined as

$$
X=\frac{x}{L}, Y=\frac{y}{L}, U=\frac{u}{u_{i}}, V=\frac{v}{u_{i}}, D=\frac{d}{L}, P=\frac{p}{\rho u_{i}^{2}}, \theta=\frac{\left(T-T_{i}\right)}{\left(T_{h}-T_{i}\right)} \text { and } \theta_{s}=\frac{\left(T_{s}-T_{i}\right)}{\left(T_{h}-T_{i}\right)}
$$

and the parameters $R e, R i, \operatorname{Pr}$ and $K$ are defined as

$$
R e=\frac{u_{i} L}{v}, R i=\frac{g \beta\left(T-T_{i}\right) L}{u_{i}^{2}}, \operatorname{Pr}=\frac{v}{\alpha} \text { and } K=\frac{k_{s}}{k}
$$

The appropriate boundary conditions (also shown in Fig. 1) used to solve the Eqs. (1) to (5) inside the cavity in dimensionless forms are given as

At the inlet: $U=1, V=0, \theta=0$

At the outlet: Convective boundary condition (CBC), $P=0$

At all solid boundaries: $U=0, V=0$

At the heated right vertical wall: $\theta=1$

At the left, top and bottom walls: $\left.\frac{\partial \theta}{\partial X}\right|_{X=0}=\left.\frac{\partial \theta}{\partial Y}\right|_{Y=1,0}=0$

At the solid-fluid vertical interfaces of the cylinder: $\left(\frac{\partial \theta}{\partial X}\right)_{\text {fluid }}=K\left(\frac{\partial \theta_{S}}{\partial X}\right)_{\text {solid }}$

At the solid-fluid horizontal interfaces of the cylinder: $\left(\frac{\partial \theta}{\partial Y}\right)_{\text {fluid }}=K\left(\frac{\partial \theta_{S}}{\partial Y}\right)_{\text {solid }}$ 
The average Nusselt number $N u$ is defined as $N u=\frac{1}{L_{h}} \int_{0}^{L_{h}} \frac{\partial \theta}{\partial X} d Y$ and the bulk average temperature in the cavity is defined as $\theta_{a v}=\int \frac{1}{\bar{V}} \theta d \bar{V}$, where $L_{h}$ is the length of the heated wall and $\bar{V}$ is the cavity volume.

\section{Numerical Technique}

The coupled governing Eqs. (1) to (5) have been solved numerically based on the Galerkin weighted residual method of finite element formulation [13, 14]. First and foremost, the solution domain is discretized into Finite element meshes, which are composed of triangular elements. Then the nonlinear governing partial differential equations, i.e., mass, momentum and energy equations are transferred into a system of integral equations by using Galerkin weighted residual. The integration involved in each term of these equations is performed with the aid of Gauss quadrature method. The nonlinear algebraic equations so obtained are modified by imposition of boundary conditions. These modified nonlinear equations are transferred into linear algebraic equations with the aid of Newton's method. Lastly, Triangular factorization method is applied for solving those linear equations. The grid refinement check and the validation of the computer code have already been verified [15].

\section{Results and Discussion}

The finite element simulation is performed to examine the laminar mixed convection flow and heat transfer in a vented square cavity having a centered horizontal solid circular cylinder. To simulate forced convection, pure mixed convection and free convection dominated flow in the enclosure, three Richardson number $R i$ (=0.0 1.0 and 5.0), respectively, has been considered. In addition, to analyze the effect of mentioned parameters on the flow and heat transfer in the cavity, the governing parameters: Reynolds number and Prandtl number has been varied from 50 to 200 and 0.71 to 7.1, respectively. The mixed convection phenomenon in the cavity is influenced by Richardson number $R i$, Reynolds number $R e$, dimensionless form of the cylinder diameter $D$ and solid fluid thermal conductivity ratio $K$. In addition, cylinder diameter and solid fluid thermal conductivity ratio are kept fixed at 0.2 and 5.0, respectively.

\subsection{Flow and thermal fields}

The effect of Reynolds number on the flow field is illustrated in Fig. 2 by plotting streamlines, while $P r=0.71$ and $R i=0.0,1.0$ and 5.0. The basic forced convection flow, presented in the left of the bottom row for $R i=0.0$ and $R e=50$, is characterized by a perfect symmetry of the solution with respect to the diagonal joining the inlet and outlet 
ports. Because of the small value of Re the thermal transport effect by the external cold air is little. Now for $R e=100$ and $R i=0.0$ it can be seen that the symmetry already observed is destroyed, and a small recirculating cell is developed just at the top of the inlet port and the recirculating cell became large with the increased of $\operatorname{Re}(150$ and 200), at the same time a small vorticity appears at the right bottom corner. Besides, at any particular Reynolds number, there is a marked changed in flow patterns as the flow regime changes from dominant forced convection to dominant natural convection with increasing $R i$.

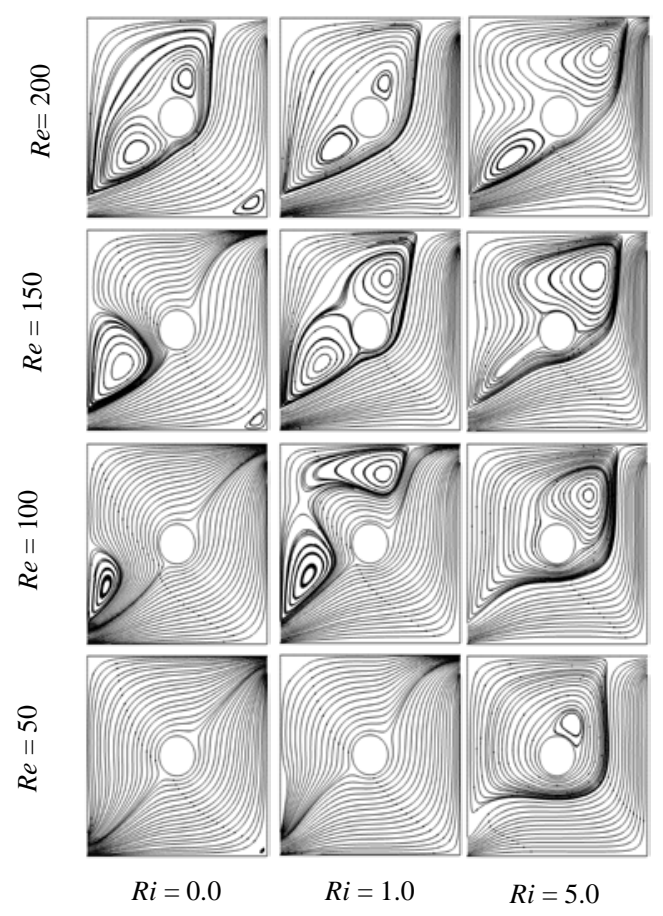

Fig. 2. Streamlines for various Reynolds numbers and $R i$, while $\operatorname{Pr}=0.71$.

The effect of Reynolds number on the temperature distributions is illustrated in Fig. 3 by plotting isotherms, while $\operatorname{Pr}=0.71$ and $R i=0.0,1.0$ and 5.0. The corresponding isotherms results from combined effects of conduction and forced convection is shown in the left bottom corner of Fig. 3, which indicated that a large area of the cavity remains at the higher temperatures. In addition, at any particular Reynolds number, there is a marked changed in isotherms as the flow regime changes from dominant forced convection to dominant natural convection with increasing $R i$. It is also observed that for a particular $R i$, the isotherms became denser toward the hot wall as the $R e$ increases. The main cause is that the region affected by the heat source became smaller due to the strong external flow. 
We now discuss the effect of Prandtl number on the flow and heat transfer in mixed convection flow on taking $R e=100$ and various values of $R i(R i=0.0,1.0$ and 5.0). Fig. 4 depicts the streamlines for the values of Prandtl number $\operatorname{Pr}=0.71,1.0,3.0$ and 7.1 at the three values of $R i$. At $R i=0.0$ and $P r=0.71$, The fluid flow is characterized by the imposed flow covering most of the cavity space and a small rotating cell is formed just at the top of the inlet port in the cavity. Moreover, the size, position and strength of the rotating cell are almost identical for the four cases presented at $R i=0.0$. It is therefore, confirmed that for $R i=1.0$, the flow in the cavity dominated by shear action of the incoming fluid, and buoyancy induced flow at low $\operatorname{Pr}=0.71$. The buoyancy induced flow become relatively weak with increasing the value of $\operatorname{Pr}$ at $R i=1.0$, since the open lines characterizing the imposed flow are still dominant. Further at $R i=5.0$ a strong effect of buoyancy forced is observed for lower values of $\operatorname{Pr}(\operatorname{Pr}=0.71$ and 1.0) and relatively weak effect of buoyancy forced is observed for higher values of $\operatorname{Pr}(\operatorname{Pr}=3.0$ and 7.1). This phenomenon is very logical because, higher Prandtl number fluids are highly viscose. The corresponding influence of Prandtl number on isotherms for different values of $R i$ is shown in Fig. 5. From this figure, we can ascertain that the isotherms are almost linear and distributed inside the cavity for $R i=0.0$ and $\operatorname{Pr}=0.71$, which is due to the combined effect of conduction and forced convection. As $R i$ increases, nonlinearity of isotherms are found and plume formation is initiated, which indicates the launch of natural convective current. An increase in Prandtl number also increases the crowding of isotherms for all values of $R i$.

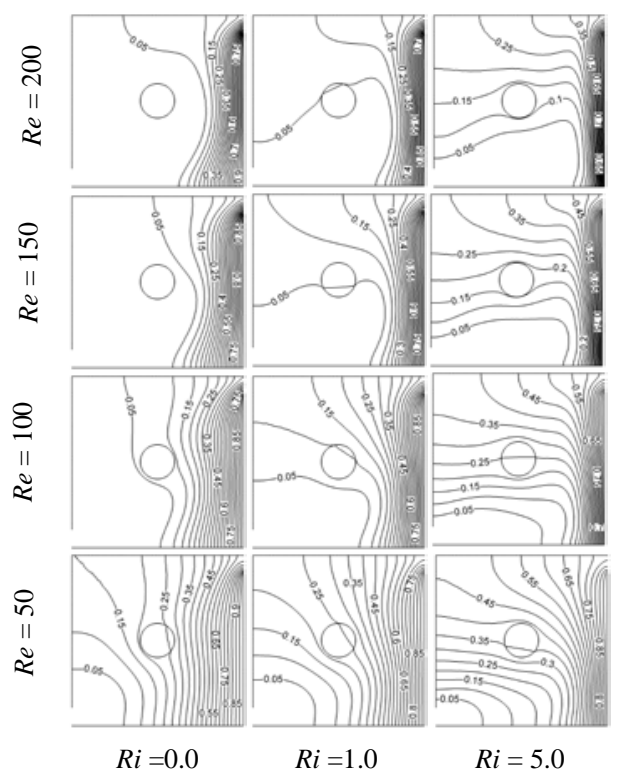

Fig. 3. Isotherms for various Reynolds numbers and $R i$, while $\operatorname{Pr}=0.71$.

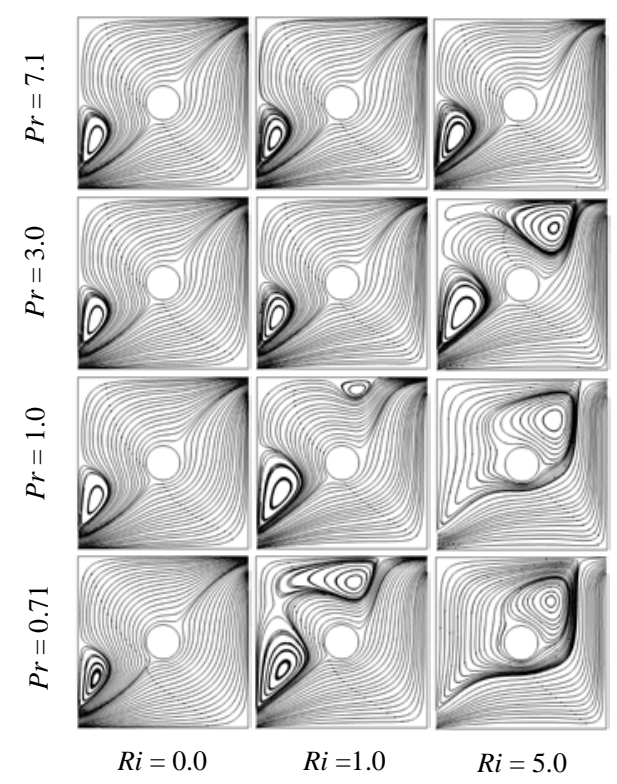

Fig. 4. Streamlines for various Prandtl numbers and $R i$, while $R e=100$. 


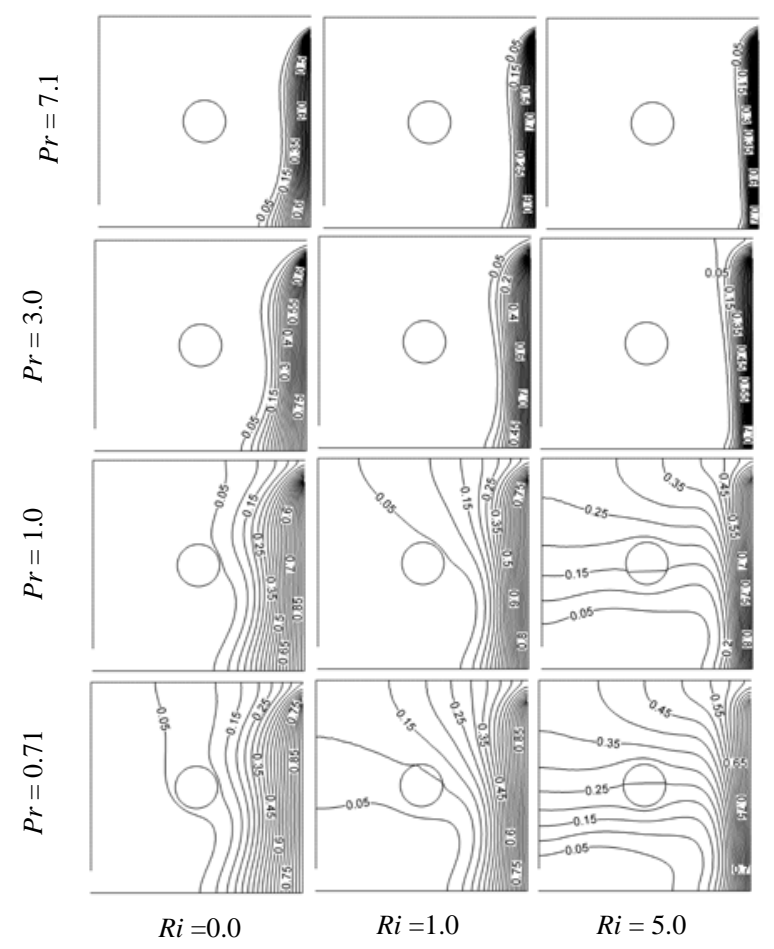

Fig.5. Isotherms for different values of Prandtl numbers and $R i$, while $R e=100$.

\subsection{Heat transfer}

The effect of Reynolds number on average Nusselt number $\mathrm{Nu}$ at the heat source, average fluid temperature $\theta_{a v}$ in the cavity and temperature $\theta_{\mathrm{c}}$ at the cylinder center is presented in Fig. 6 as a function of Richardson number. Keeping the values of $R e$ constant, the average Nusselt number at the heated surface increases gradually with increasing values of $R i$. Also, the average Nusselt number at the heated surface is found to increase as $R e$ increases at fixed Ri. Therefore, it can be concluded that more heat transfer from the heat source is expected in the case of large parameter value of $R e$ or $R i$. The average temperature of the fluid domain and temperature at the cylinder center in the cavity increases gradually with $R i$ for all values of $R e$. Moreover, for a fixed value of $R i$, the values of $\theta_{a v}$ and $\theta_{c}$ are the lowest for the largest $R e$.

The effect of Prandtl number on average Nusselt number $N u$ at the heated surface, average temperature $\theta_{a v}$ of the fluid in the cavity and temperature $\theta_{\mathrm{c}}$ at the cylinder center as a function of Richardson number is shown in Fig. 7. As $R i$ increases, average Nusselt number $\mathrm{Nu}$ increases monotonically for all values of $\mathrm{Pr}$. Maximum average Nusselt number is obtained, without the effect of $R i$, for the highest $\operatorname{Pr}=7.1$. The average temperature of the fluid domain and the temperature at the cylinder center in the cavity increases gradually with the increases of $R i$ for the lower values of $\operatorname{Pr}(=0.71$ and 1.0 ), 
while the average temperature of the fluid decreases gradually with increasing $R i$ for the higher values of $\operatorname{Pr}$ (= 3.0 and 7.1). On the other hand, the temperature at the cylinder center is independent of $R i$ for the higher values of $\operatorname{Pr}$ (= 3.0 and 7.1). However, minimum average temperature of the fluid and the temperature at the cylinder center in the cavity are obtained, without the effect of $R i$, for the highest $\operatorname{Pr}=7.1$.
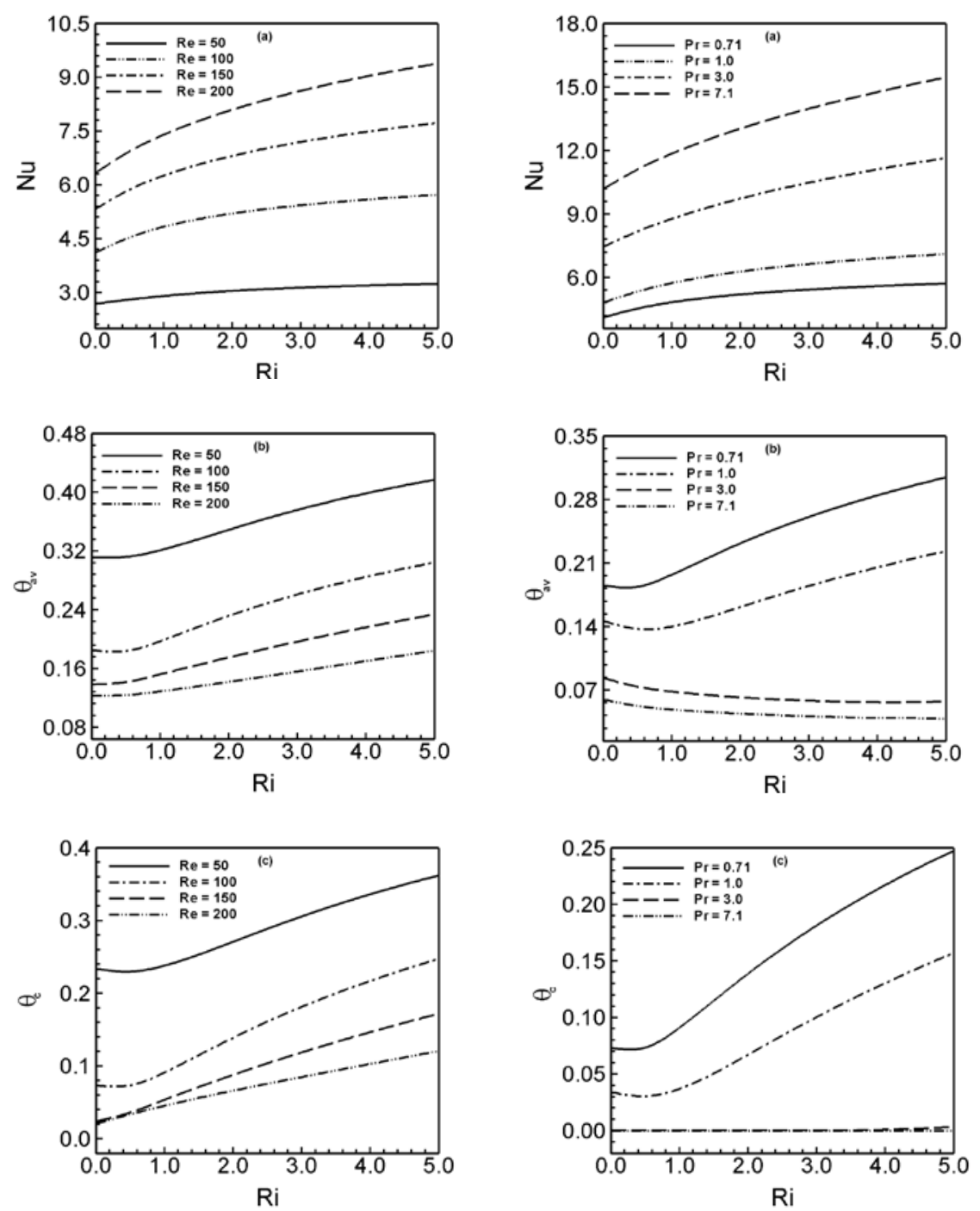

Fig. 6. Effect of Re on (a) average Nusselt number, (b) average fluid temperature and (c) temperature at the cylinder center in the cavity, while $\operatorname{Pr}=0.71$.

Fig.7. Effect of $\operatorname{Pr}$ on (a) average Nusselt number, (b) average fluid temperature and (c) temperature at the cylinder center in the cavity, while $R e=100$. 
The effect of Prandtl number on average Nusselt number $\mathrm{Nu}$ at the heated surface, average temperature $\theta_{a v}$ of the fluid in the cavity and temperature $\theta_{\mathrm{c}}$ at the cylinder center as a function of Richardson number is shown in Fig. 7. As Ri increases, average Nusselt number $\mathrm{Nu}$ increases monotonically for all values of $\mathrm{Pr}$. Maximum average Nusselt number is obtained, without the effect of $R i$, for the highest $\operatorname{Pr}=7.1$. The average temperature of the fluid domain and the temperature at the cylinder center in the cavity increases gradually with the increases of $R i$ for the lower values of $\operatorname{Pr}(=0.71$ and 1.0 ), while the average temperature of the fluid decreases gradually with increasing $R i$ for the higher values of $\operatorname{Pr}$ (= 3.0 and 7.1). On the other hand, the temperature at the cylinder center is independent of $R i$ for the higher values of $\operatorname{Pr}$ (= 3.0 and 7.1). However, minimum average temperature of the fluid and the temperature at the cylinder center in the cavity are obtained, without the effect of $R i$, for the highest $\operatorname{Pr}=7.1$.

\section{Conclusion}

A numerical investigation of mixed convection in a ventilated square cavity with a heat conducting horizontal solid circular cylinder has been performed. Results have been presented in terms of streamlines, isotherms, average Nusselt number at the heated surface average temperature of the fluid and the temperature at the cylinder center to analyze the effect of the Reynolds number and Prandtl number on the fluid flow and heat transfer in the cavity for the aforementioned Richardson numbers. In view of obtained results, the following findings have been summarized:

a. Reynolds number $R e$ has a great significant effect on the streamlines and isotherms at the three convective regimes. Buoyancy-induced vortex in the streamlines increased and thermal layer near the heated surface become thin and concentrated with increasing $R e$. The average Nusselt numbers at the heated surface is always upper and the average temperature in the cavity is inferior for the largest value of $R e$.

b. Recirculation cell in the streamline plot decreases and thermal boundary layer thickness near the heated wall decreases strongly with increasing the values of $\mathrm{Pr}$ at the three convective regimes. Maximum average Nusselt number, minimum average fluid temperature and cylinder center temperature are obtained, without the effect of $R i$, for the highest $\operatorname{Pr}=7.1$.

\section{Acknowledgements}

The authors would like to express their gratitude to the Department of Mathematics, Bangladesh University of Engineering and Technology, for providing computer facility during this work.

\section{References}

1. J. M. House, C. Beckerman and T. F. Smith, Numer. Heat Transfer (Part A) 18, 213 (1990). doi:10.1080/10407789008944791 
2. J. Y. Oh, M. Y. Ha and K. C. Kim, Numer. Heat Transfer (Part A) 31, 289 (1997). doi:10.1080/10407789708914038

3. D. G. Roychowdhury, S. K. Das and T. S. Sundararajan, Heat and Mass Transfer 38, 565 (2002). doi:10.1007/s002310100210

4. S. F. Dong and Y.T. Li, Int. J. of Heat and Mass Transfer 47, 2233 (2004). doi:10.1016/j.ijheatmasstransfer.2003.11.018

5. E. Bilgen and T. Yamane, Heat and Mass Transfer 40, 401 (2004). doi:10.1007/s00231-003-0418-z

6. M. T. Bhoite, G. S. V. L Narasimham and M. V. K. Murthy, Int. J. of Thermal Sciences 44, 125 (2005). doi:10.1016/j.ijthermalsci.2004.07.003

7. E. J. Braga and M. J. S. de Lemos, Int. Commun. Heat Mass Transfer 32, 1289 (2005). doi:10.1016/j.icheatmasstransfer.2005.07.014

8. S. H. Tasnim and M. R. Collins, Int. Commun. Heat Mass Transfer 32, 94 (2005). doi:10.1016/j.icheatmasstransfer.2004.05.022

9. M. K. Das and K. S. K. Reddy, Int. J. of Heat and Mass Transfer 49, 4987 (2006). doi:10.1016/j.ijheatmasstransfer.2006.02.050

10. F. Xu, J. C. Patterson and C. Lei, Experiments in Fluids 40, 364 (2006). doi:10.1007/s00348-005-0073-x

11. F. Y. Zhao, G. F. Tang and D. Lin, Int. J. of Engg. Science 44, 148 (2006). doi:10.1016/j.ijengsci.2005.10.006

12. M. M. Rahman, Ph.D. Thesis, Bangladesh University of Engineering and Technology, Dhaka, Bangladesh (2009).

13. C. Taylor and P. Hood, Computer and Fluids 1, 73 (1973). doi:10.1016/0045-7930(73)90027-3

14. P. Dechaumphai, Finite Element Method in Engineering, 2nd Edition, (Chulalongkorn University Press, Bangkok, 1999).

15. M. M. Rahman, M. A. Alim, S. Saha and M. K. Chowdhury, J. of Naval Architecture and Marine Engg. 2, 37 (2008). 